\title{
New Semiclassical Picture of Vacuum Decay
}

\author{
Jonathan Braden, ${ }^{1,}{ }^{*}$ Matthew C. Johnson, ${ }^{2}$ Hiranya V. Peiris, ${ }^{1,3}$ Andrew Pontzen, ${ }^{1}$ and Silke Weinfurtner ${ }^{4}$ \\ ${ }^{1}$ Department of Physics and Astronomy, University College London, Gower Street, London, WC1E 6BT, United Kingdom \\ ${ }^{2}$ Department of Physics and Astronomy, York University, Toronto, Ontario, M3J 1P3, Canada \\ and Perimeter Institute for Theoretical Physics, 31 Caroline Street N, Waterloo, Ontario, N2L 2Y5, Canada \\ ${ }^{3}$ The Oskar Klein Centre for Cosmoparticle Physics, Stockholm University, AlbaNova, Stockholm, SE-106 91, Sweden \\ ${ }^{4}$ School of Mathematical Sciences, University of Nottingham, University Park, Nottingham, NG7 2RD, United Kingdom \\ and Centre for the Mathematics and Theoretical Physics of Quantum Non-Equilibrium Systems, \\ University of Nottingham, Nottingham, NG7 2RD, United Kingdom
}

(Received 29 June 2018; revised manuscript received 31 May 2019; published 18 July 2019)

\begin{abstract}
We introduce a new picture of vacuum decay which, in contrast to existing semiclassical techniques, provides a real-time description and does not rely on classically forbidden tunneling paths. Using lattice simulations, we observe vacuum decay via bubble formation by generating realizations of vacuum fluctuations and evolving with the classical equations of motion. The decay rate obtained from an ensemble of simulations is in excellent agreement with existing techniques. Future applications include bubble correlation functions, fast decay rates, and decay of nonvacuum states.
\end{abstract}

DOI: 10.1103/PhysRevLett.123.031601

Introduction.-Field theories with complex interaction potentials arise in a diverse range of physical applications: from cosmological theories of the multiverse inspired by string theory [1,2], to cold atom Bose-Einstein condensates (BECs) [3-5], to the dynamics of protein folding [6]. Such theories generically possess many local potential minima. At the homogeneous classical level such local potential minima are stable. However, the uncertainty principle ensures that spatially inhomogeneous quantum fluctuations are present, resulting in the metastablility of false vacuum field configurations. The decay of false vacuum states is expected to proceed analogously to first-order phase transitions driven by statistical fluctuations in condensed matter systems $[7,8]$, with bubbles of a new phase nucleating then expanding into the ambient false vacuum. The prevailing, if implicit, wisdom is that bubbles nucleate via nonclassical paths in field space, analogous to tunneling through a barrier in quantum mechanics [9-16]. We propose an alternative picture of vacuum decay, in which the classical evolution of the field from some initial realization of the false vacuum fluctuations leads to the emergence of bubbles. As convenient nomenclature, we will refer to these as classically forbidden and classically allowed nucleations, respectively.

We reexamine the nature of bubble nucleations during false vacuum decay in relativistic scalar field theory using

Published by the American Physical Society under the terms of the Creative Commons Attribution 4.0 International license. Further distribution of this work must maintain attribution to the author(s) and the published article's title, journal citation, and DOI. Funded by SCOAP ${ }^{3}$. semiclassical scalar field lattice simulations. In our framework, quantum effects are included by sampling realizations of the initial quantum state of the field, then time evolving using the classical equations of motion. As we demonstrate in the Supplemental Material [17], this captures the time evolution of the wave functional to $\mathcal{O}(\hbar)$ (see also Ref. [18] for a similar derivation). This treatment is analogous to the truncated Wigner approximation [19,20] often employed in atomic physics. We explicitly demonstrate the existence of classically allowed paths connecting these initial realizations of the quantum false vacuum to a subsequent state with nucleated bubbles of "true vacuum." This demonstrates that false vacuum decay can proceed via classically allowed dynamical evolution. Furthermore, we compare the nucleation rates inferred using classical statistics for the initial fluctuations (i.e., ignoring quantum interference), and find good agreement with instantonbased predictions which cannot tackle the time-dependent nature of the problem. This suggests that the decay of the false vacuum proceeds via classically allowed bubble nucleation events. The Supplemental Material shows how this approach can be interpreted as an expansion of the full quantum dynamics in $\hbar$ [17].

In this work, we focus on the particular field theory emerging from the cold atom analog false vacuum proposal of Fialko et al. [3,4] (see also Braden et al. [5]). However, we do not expect the presence of classically allowed decay channels to depend strongly on the specific choice of field theory. The possible connection to experiment is particularly exciting in this context, as it provides a window to experimentally confirm the dynamics of vacuum decay, and possibly uncover additional effects arising from quantum interference such as Anderson localization [21,22]. 
Semiclassical statistical formalism.-We study relativistic false vacuum decay using semiclassical statistical lattice simulations. Throughout, we work in units with $\hbar=c=1$. In order to generate sufficient statistics to compute decay rates, we will work with fields in $1+1$ dimensions, although we do not expect consideration of higher spatial dimensions to qualitatively change our conclusions.

The fields are initialized as

$$
\phi(\mathbf{x}, t=0)=\phi_{\mathrm{fv}}+\delta \hat{\phi}(\mathbf{x}), \quad \dot{\phi}(\mathbf{x}, t=0)=\delta \hat{\dot{\phi}}(\mathbf{x}),
$$

where the fluctuations $\delta \hat{\phi}$ and $\delta \hat{\dot{\phi}}$ are realizations of random fields with the same statistics as the quantum Minkowski vacuum fluctuations, and $\phi_{\mathrm{fv}}$ is the mean field value in the false vacuum. Using these as initial conditions, we solve Hamilton's equations,

$$
\frac{d \phi}{d t}=\dot{\phi} \quad \text { and } \quad \frac{d \dot{\phi}}{d t}=\nabla^{2} \phi-V^{\prime}(\phi),
$$

with a tenth-order accurate Gauss-Legendre time integrator $[23,24]$. The spatial Laplacian is computed using a Fourier collocation stencil, resulting in periodic boundary conditions. This combination conserves energy to $\mathcal{O}\left(10^{-15}\right)$ with a maximal pointwise error of $\mathcal{O}\left(10^{-13}\right)$ as either the grid spacing or time step is varied. Additionally, our time integrator is symplectic so that phase space volume is preserved. Finally, we verified that our numerical simulations are time reversible. First we integrate forward in time well into the regime where the false vacuum has decayed completely. By reversing the flow of time and backward integrating for an equal time interval, we are able to recover the initial state of $\phi$ and $\dot{\phi}$ to a precision of $\mathcal{O}\left(10^{-15}\right)$. Further, if we continue to backward integrate in time, we again see the initial state decay since the false vacuum is not a true eigenstate of the Hamiltonian.

This approach does not capture quantum interference or the contributions of nonclassical paths, which, as we demonstrate in the Supplemental Material [17], enter at $\mathcal{O}\left(\hbar^{2}\right)$ in our approach. Nonetheless, there are many cases in which this semiclassical approach gives an accurate description of the statistics of the field(s). Some wellknown cases include postinflation preheating dynamics and relativistic heavy ion collisions [25-29]. In these standard scenarios, individual Fourier modes become highly squeezed while in the linear regime, thus entering the classical wave limit and justifying a classical treatment of the field dynamics and statistics. The case considered here is novel, since once a bubble has formed in the medium the fluctuations are no longer statistically homogeneous, and the growth of modes leading to its formation may not occur in the linear regime. However, a bubble is a highly classical field configuration, so we expect that many aspects will be properly captured by our simulations. Regardless of how accurately these semiclassical simulations capture the full quantum statistics, they are more than sufficient to demonstrate the existence of classically allowed bubble nucleation trajectories.

Classically allowed decays.-We now use the framework outlined above to study the dynamics of a scalar field $\phi$ initially trapped in a false vacuum. Motivated by the analog false vacuum setup in cold atom Bose-Einstein condensate systems [3-5], we consider the following action:

$$
\mathcal{L}_{\phi}=\frac{\dot{\phi}^{2}}{2}-\frac{(\nabla \phi)^{2}}{2}-V_{0}\left(-\cos \frac{\phi}{\phi_{0}}+\frac{\lambda^{2}}{2} \sin ^{2} \frac{\phi}{\phi_{0}}\right) .
$$

In the BEC experiments, $\lambda$ is an experimentally tunable parameter controlling the depth of the false vacuum well. For $\lambda>1$, this theory possesses an infinite sequence of false vacuum extrema at $\phi=(2 k+1) \pi$, with $k \in \mathbb{Z}$. Instead, for $\lambda<1$ the false vacua are local maxima of the potential, and states centered around these points undergo spinodal decay.

Here, we approximate the initial vacuum fluctuations as those of a free massive scalar field with mass squared $m_{\text {eff }}^{2}=V^{\prime \prime}\left(\phi=\pi \phi_{0}\right)=V_{0} \phi_{0}^{-2}\left(-1+\lambda^{2}\right)$. We sample fluctuations of the field $\delta \phi$ and its time derivative $\delta \dot{\phi}$ as uncorrelated Gaussian random fields with spectra

$$
\left\langle\phi_{k}^{*} \phi_{k^{\prime}}\right\rangle=\frac{1}{2 \omega_{k}} \delta\left(k-k^{\prime}\right), \quad\left\langle\dot{\phi}_{k}^{*} \dot{\phi}_{k^{\prime}}\right\rangle=\frac{\omega_{k}}{2} \delta\left(k-k^{\prime}\right),
$$

where $\omega_{k}^{2}=k^{2}+m_{\mathrm{eff}}^{2}$ and $\delta\left(k-k^{\prime}\right)$ is the Dirac delta function.

It is convenient to work with the dimensionless variable $\bar{\phi}=\phi / \phi_{0}$, along with dimensionless units $\bar{t}=\mu t$ and $\bar{x}=\mu x$, where $\mu$ is a constant with dimensions of mass. Explicitly, for a one-dimensional discrete lattice of finite size $L$,

$$
\delta \hat{\bar{\phi}}(\mathbf{x})=\frac{1}{\phi_{0}} \frac{1}{\sqrt{2 L}} \sum_{n=1}^{n_{\mathrm{cut}}} e^{i \mathbf{k}_{n} \cdot \mathbf{x}} \frac{\hat{\alpha}_{n}}{\left(m_{\mathrm{eff}}^{2}+k_{n}^{2}\right)^{1 / 4}},
$$

where $\hat{\alpha}_{n}$ are realizations of complex Gaussian random deviates with unit variance, which we generate using $\hat{\alpha}_{n}=$ $\sqrt{-\ln \hat{a}_{n}} e^{i 2 \pi \hat{b}_{n}}$, where $\hat{a}_{n}$ and $\hat{b}_{n}$ are uniform random deviates on the unit interval. We have included a sharp cutoff in the spectrum, which must be less than or equal to the Nyquist frequency, and the wave vectors are given by $k_{n}=$ $(2 \pi n / L)$. The $\delta \dot{\phi}$ fluctuations are initialized analogously.

Figure 1 shows an example classical field evolution in one spatial dimension for a single initial field realization described above. We see a bubble (i.e., domain wall-antiwall pair) nucleate from the initial vacuum fluctuations, then subsequently expand into the surrounding false vacuum. 


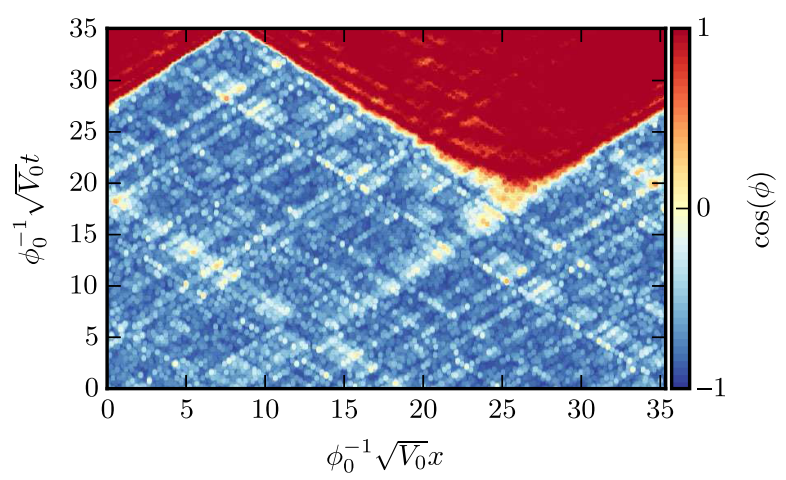

FIG. 1. A classically allowed false vacuum decay for the Lagrangian density Eq. (3) with $\lambda=1.2$ and $\phi_{0}=(\pi / 4 \sqrt{2})$. The initial fluctuations $\delta \phi$ and $\delta \dot{\phi}$ are realizations of Gaussian random fields with spectra mimicking Minkowski false vacuum fluctuations. We observe the dynamical emergence of a bubble with field localized near a true vacuum $(\cos \phi=1)$ embedded within the false vacuum $(\cos \phi=-1)$. This explicitly demonstrates the existence of classically allowed paths for false vacuum decay.

To our knowledge, this is the first explicit demonstration that bubble nucleation in false vacuum decay can occur via classically allowed field evolutions in pure relativistic field theory. Aside from the nature of quantum versus classical statistics, this is analogous to bubble formation in thermal [30] (or other classical statistical [31]) phase transitions. Thermal nucleation events are also typically modeled as random, but at a microscopic level the full set of field fluctuations (including the "thermal bath") are simply following the classical equations of motion. Bubble nucleations are special classical trajectories through phase space that describe the coherent creation of a bubble. In the quantum case we are modeling, the fluctuations instead descend directly from the initial state of the field rather than an external bath.

Let us now consider how to interpret the results of our simulations. A complete time-dependent description requires obtaining the wave functional

$$
\langle\phi(\mathbf{x}) \mid \Psi(t)\rangle=\int \mathcal{D} \phi_{i}\left\langle\phi\left|\hat{U}\left(t \mid t_{0}\right)\right| \phi_{i}\right\rangle\left\langle\phi_{i}(\mathbf{x}) \mid \Psi\left(t_{0}\right)\right\rangle
$$

for the full scalar field configuration as a function of time, given the initial wave functional $\left|\Psi\left(t_{0}\right)\right\rangle$. However, any observer watching the false vacuum boil will not have access to the full quantum state of the field as encoded in $|\Psi(t)\rangle$. Rather than seeing a quantum superposition of nucleation events, such an observer will see $N_{b}$ bubbles nucleate with spacetime locations $\left\{\left(\mathbf{x}_{i}, t_{i}\right), i=1, N_{b}\right\}$. A priori, we can only predict probabilities for $N_{b}$ and these spacetime positions. Given that an actual set of nucleation events has taken place, the observer should be able to describe the field evolution subject to the constraint of these observed nucleations. Since the observer will not have perfect knowledge of the multibubble state, their observations should be marginalized over fluctuations around the constrained field path. The result presented here shows that there exist constrained paths describing purely classical time evolution connecting final multibubble states directly to realizations of the initial vacuum fluctuations. Since these classical paths extremize the action, we expect that they will provide an important contribution to the propagator determining the evolution of the wave functional.

To contrast our approach, let us instead briefly consider the standard Euclidean (i.e., instanton) formalism $[9,10,14]$. We Wick rotate to Euclidean time, then search for extremal paths of the Euclidean action connecting the false vacuum at infinity to the true vacuum at the origin. These extremal paths are known as bounce solutions. Nearly all existing treatments assume the bounce is spherically symmetric in Euclidean signature. This dramatically reduces the phase space of allowed field configurations, simplifying it to a quantum mechanics problem. Nearly all existing treatments assume the bounce is spherically symmetric. In Lorentzian signature, the bounce describes a spherically symmetric bubble that contracts from infinite size in the distant past, reaches a minimum turnaround radius, and then reexpands. The nucleation event itself is modeled by artificially removing the contracting phase, then pasting the remaining expanding phase into the ambient spacetime. The pasting step is interpreted as a classically forbidden quantum tunneling process, analogous to the decay of an unstable particle in quantum mechanics.

However, the tunneling interpretation is fueled by analogy with quantum mechanics, which, as mentioned above, requires a drastic reduction in the allowed phase space for the field evolution. We have demonstrated that the neglected fluctuations can play a pivotal role around the time of nucleation, and allow for bubbles to nucleate via classically allowed trajectories. Specific fluctuation configurations that may lead to bubble formation are (1) local peaks or valleys in the initial fluctuations around the false vacuum that probe the nonlinear structure of the potential and act as bubble nucleation seeds or (2) the repeated mildly nonlinear interactions of propagating waves that eventually lead to the development of a local field peak from which a bubble may form. The latter effect means that energy within a small region may not be conserved due to energy transport via currents.

False vacuum decay rates. - Thus far, we showed the existence of classically allowed paths through field space describing false vacuum decay via bubble nucleation. Given that such paths exist, we expect they play an important part in the false vacuum decay process. They may either be a competing process with classically forbidden bubble nucleations or, given the drastic dimensional reduction used in the Euclidean formalism, may actually be a more complete description of the decays captured by instanton techniques. To investigate this issue, we estimate 
the bubble nucleation rate using our real-time simulations and compare it with the instanton predictions.

In $D=d+1$ spacetime dimensions, the Euclidean prediction for the nucleation rate per unit volume is

$$
\begin{aligned}
\frac{\Gamma}{V} & =\left(\frac{B}{2 \pi}\right)^{D / 2} e^{-B}\left(\frac{\operatorname{det}^{\prime} \delta^{2} S_{E}\left[\phi_{B}\right]}{\operatorname{det} \delta^{2} S_{E}\left[\phi_{\mathrm{fv}}\right]}\right)^{-1 / 2} \\
& \equiv\left(\frac{B}{2 \pi}\right)^{D / 2} e^{-B} D\left(\lambda, \phi_{0}\right),
\end{aligned}
$$

where $B=S_{E}\left[\phi_{B}\right]-S_{E}\left[\phi_{\mathrm{fv}}\right]$ is the difference in the Euclidean action evaluated on the bounce solution and in the false vacuum, det' indicates the determinant with zero modes removed, and $\delta^{2} S_{E}$ is the second variation of the Euclidean action. The bounce $\phi_{B}$ satisfies

$$
\frac{\partial^{2} \phi_{B}}{\partial r_{E}^{2}}+\frac{d}{r_{E}} \frac{\partial \phi_{B}}{\partial r_{E}}-\frac{\partial V}{\partial \phi}=0,
$$

with boundary conditions $\phi_{B}\left(r_{E}=\infty\right)=\phi_{\mathrm{fv}}$ and $\partial_{r_{E}} \phi$ $\left(r_{E}=0\right)=0$. In addition, $\phi_{B}\left(r_{E}=0\right)$ should be near the true vacuum, and the solution should possess a single negative eigenmode to give an imaginary contribution to the Euclidean effective action [32]. A precise calculation of the determinant prefactor is beyond the scope of this Letter, so we parametrize it as $D\left(\lambda, \phi_{0}\right)=g\left(\lambda, \phi_{0}\right)\left(V_{0} \phi_{0}^{-2}\right)^{D / 2}$, where $g$ is a dimensionless function of order unity which includes counterterms to renormalize the determinant. (When the bounce solution possesses a hierarchy of scales, such as in the thin-wall limit, $g$ may not be order one.) For the purpose of obtaining scaling relations, it is convenient to reexpress Eq. (7) as

$$
\frac{\bar{\Gamma}}{\bar{L}} \equiv \frac{1}{\mu^{2}} \frac{\Gamma}{L}=2 g\left(\lambda, \phi_{0}\right)\left(\frac{V_{0}}{\mu^{2} \phi_{0}^{2}}\right) \phi_{0}^{2} C(\lambda) e^{-2 \pi \phi_{0}^{2} C(\lambda)},
$$

where $C(\lambda)=\int \bar{r}_{E} d \bar{r}_{E}\left\{\left[\left(\partial_{\bar{r}_{E}} \bar{\phi}_{B}\right)^{2} / 2\right]+\left(1 / \mu^{2} \phi_{0}^{2}\right)[V(\phi)-\right.$ $\left.\left.V\left(\phi_{\mathrm{fv}}\right)\right]\right\}$ and we have specialized to one spatial dimension. For our particular model, $V(\phi)=V_{0}\left[-\cos \phi+\left(\lambda^{2} / 2\right)\right.$ $\left.\sin ^{2} \phi\right]$. The factor of 2 accounts for the independent instanton decay paths in this model.

As is clear from Fig. 1, the emergence of a bubble is a "fuzzy" process which makes a precise determination of the nucleation time somewhat ambiguous. We therefore adopt the following operational definition. Imagine an ensemble of observers each watching a finite region of the false vacuum boil. We ask each of these observers to provide us with a history of $\langle\cos \phi\rangle_{V}$ within their region, where $\langle\cdot\rangle_{V}$ represents a spatial average. To obtain statistics, we make one additional assumption-each field trajectory (and thus each observer) is weighted by the probability of the initial realization of the fluctuations. In the particular case considered here, where we assume the initial state is Gaussian and draw samples from an ensemble of Gaussian random fields, each simulation is weighted equally. We select a threshold,

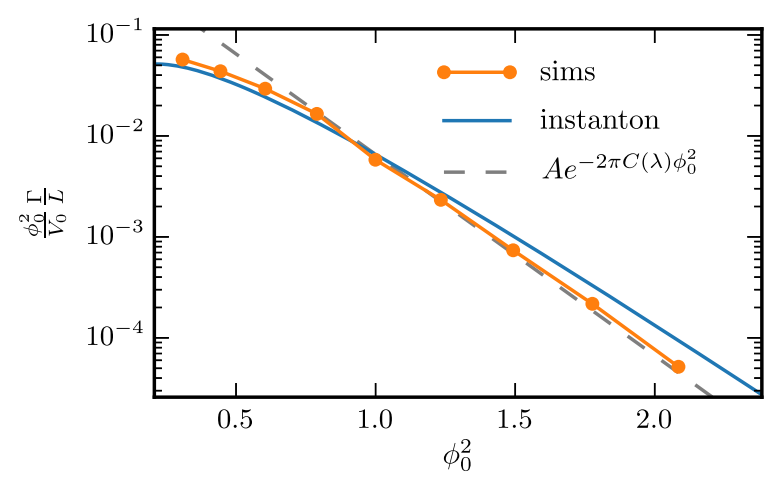

FIG. 2. Decay rates of false vacuum initial states as $\phi_{0}$ is varied for our ensembles of lattice simulations (orange dots). For comparison, the blue solid line is the analytic prediction Eq. (9) with $g=-1+\lambda^{2}=0.44$ to set the overall scale to the false vacuum mass, and the gray dashed line is the leading-order prediction (i.e., $\Gamma=A e^{-2 \pi C(\lambda) \phi_{0}^{2}}$ with the constant $A$ normalized so the curves match at $\left.\phi_{0}=1\right)$. We fixed $\lambda=1.2$ and $\left(V_{0} / \phi_{0}^{2} \mu^{2}\right)=$ $8 \times 10^{-3}$.

and declare that the observer's region has decayed at the first passage of $\langle\cos \phi\rangle_{V}$ past this threshold. To reduce the effects of multiple bubbles and fluctuations, we choose the threshold to be $\bar{c}_{T}+n_{\sigma} \Delta c_{T}$ for some constant $n_{\sigma}$. Here, $\bar{c}_{T}$ and $\Delta c_{T}$ are, respectively, the ensemble average and standard deviation of $\langle\cos \phi\rangle_{V}$ on the initial time slice. We then run ensembles of 5000 lattice simulations with varying $\phi_{0}$ and side length $L$ for $\lambda=1.2$, and empirically determine the fraction of undecayed trajectories $F_{\text {survive }}(t)$. We verified $F_{\text {survive }}$ is insensitive to the choices of both the lattice spacing $d x$ and the threshold $n_{\sigma}$ for $5<n_{\sigma}<25$. After an initial transient that depends on the simulation volume $L, F_{\text {survive }}$ enters a regime of nearly exponential decay. We fit this exponential tail $F_{\text {survive }}=e^{-\Gamma\left(t-t_{0}\right)}$ with parameters $\Gamma$ and $t_{0}$ to extract the decay rate and verified that $\Gamma / L$ is independent of the simulation volume $L$.

In Fig. 2 we compare the decay rates extracted from our simulations with those of the instanton formalism (obtained using the method of Ref. [33]). We also include the leading order prediction to compare to an exponential falloff at large $\phi_{0}$. To capture all relevant dynamical modes, we set the spectral cutoff $k_{\text {cut }}=(1024 \pi / 25 \sqrt{2})\left(\sqrt{V_{0}} / \phi_{0}\right)$ above the wave number where the Fourier amplitude of the bounce profile $\phi_{B}$ dropped below $10^{-15}$. In this regime, increasing $k_{\text {cut }}$ should simply renormalize the field theory. Given the unknown determinant prefactor, exponential sensitivity of the decay rate to the bounce action, and multibubble and fluctuation effects entering into our decay time extraction, our simulations match the instanton prediction very well. In particular, the amplitude is the correct order of magnitude, and the rate of exponential decay at large $\phi_{0}$ closely matches the Euclidean calculation.

We also varied $\lambda$ at fixed $\phi_{0}^{2} C(\lambda)$ and found the time evolution of the survival fraction to be nearly independent 
of the choice of $\lambda$. This suggests not only that classically allowed decay paths are a key process in false vacuum decay, but also that these classically allowed paths may be the same ones being captured by the standard instanton calculation. In order to address this latter issue, the results presented here motivate a deeper investigation of the function $g$, renormalization effects, and a more sophisticated bubble extraction algorithm.

Conclusions. - We outlined a novel real-time framework to study quantum false vacuum decay, based on semiclassical stochastic lattice simulations. This provides a step towards incorporating the full temporal evolution of a decaying region of the false vacuum. In particular, we demonstrated that the false vacuum can decay via bubble nucleation along classically allowed field trajectories, and the decay rates of such decays agree well with standard computations based on the instanton formalism. This suggests that our formalism provides a description of the "fuzzy" process of a bubble nucleating in false vacuum. We will explore how our interpretation generalizes to other systems of nonequilibrium bubble formation in a forthcoming work.

One particularly exciting prospect is the possibility of emulating the false vacuum decay process with cold atom systems, suggesting we may be able to experimentally test the picture outlined here [3-5]. In addition, our approach opens new avenues of investigation which cannot be addressed within the Euclidean framework. These include decay rates from nonvacuum initial states, investigation of bubble-bubble correlations, and evolution in a regime of rapid decays. Furthermore, the emergence of a classical bubble from a highly quantum initial state is an example of self-decoherence of a quantum field, and may yield insight into the process by which effectively classical density perturbations arise from quantum fluctuations during inflation [34-37]. Finally, the emergence of a classical bubble from the quantum vacuum bears many similarities to the process of "measuring" a bubble nucleation event, and may shed light on the notion of measurement in quantum field theory and quantum cosmology [38].

J. B. and H. V.P. were supported by the European Research Council (ERC) under the European Community's Seventh Framework Programme (FP7/20072013)/ERC Grant Agreement No. 306478-CosmicDawn. M.C.J. was supported by the National Science and Engineering Research Council through a Discovery grant. A. P. was supported by the Royal Society. S. W. acknowledges financial support provided under the Royal Society University Research Fellow (UF120112), the Nottingham Advanced Research Fellow (A2RHS2), the Royal Society Project (RG130377) grants, and the EPSRC Project Grant (EP/P00637X/1). This work was partially enabled by funding from the UCL Cosmoparticle Initiative. This research was supported in part by Perimeter Institute for Theoretical Physics. Research at Perimeter Institute is supported by the Government of Canada through the Department of
Innovation, Science and Economic Development Canada and by the Province of Ontario through the Ministry of Research, Innovation and Science.

* Corresponding author.

jbraden@cita.utoronto.ca

[1] R. Bousso and J. Polchinski, Quantization of four form fluxes and dynamical neutralization of the cosmological constant, J. High Energy Phys. 06 (2000) 006.

[2] L. Susskind, The anthropic landscape of string theory, in Universe or Multiverse, edited by B. Carr (Cambridge University Press, Cambridge, England, 2007), pp. 247-266.

[3] O. Fialko, B. Opanchuk, A. I. Sidorov, P. D. Drummond, and J. Brand, Fate of the false vacuum: towards realization with ultra-cold atoms, Europhys. Lett. 110, 56001 (2015).

[4] O. Fialko, B. Opanchuk, A. I. Sidorov, P. D. Drummond, and J. Brand, , The universe on a table top: engineering quantum decay of a relativistic scalar field from a metastable vacuum, J. Phys. B 50, 024003 (2017).

[5] J. Braden, M. C. Johnson, H. V. Peiris, and S. Weinfurtner, Towards the cold atom analog false vacuum, J. High Energy Phys. 07 (2018) 014.

[6] J. N. Onuchic, Z. Luthey-Schulten, and P. G. Wolynes, Theory of protein folding: The energy landscape perspective, Annu. Rev. Phys. Chem. 48, 545 (1997).

[7] J. S. Langer, Theory of the condensation point, Ann. Phys. (N.Y.) 41, 108 (1967); 281, 941 (2000).

[8] J. S. Langer, Statistical theory of the decay of metastable states, Ann. Phys. (N.Y.) 54, 258 (1969).

[9] S. R. Coleman, The fate of the false vacuum: Semiclassical theory, Phys. Rev. D 15, 2929 (1977); Erratum, Phys. Rev. D 16, 1248(E) (1977).

[10] C. G. Callan, Jr. and S. R. Coleman, Fate of the false vacuum. II. first quantum corrections, Phys. Rev. D 16, 1762 (1977).

[11] S. R. Coleman, V. Glaser, and A. Martin, Action minima among solutions to a class of euclidean scalar field equations, Commun. Math. Phys. 58, 211 (1978).

[12] K.-M. Lee and E. J. Weinberg, Tunneling without barriers, Nucl. Phys. B267, 181 (1986).

[13] C. L. Wainwright, CosmoTransitions: Computing cosmological phase transition temperatures and bubble profiles with multiple fields, Comput. Phys. Commun. 183, 2006 (2012).

[14] E. J. Weinberg, Classical Solutions in Quantum Field Theory, Cambridge Monographs on Mathematical Physics (Cambridge University Press, Cambridge, England, 2012).

[15] B. Greene, D. Kagan, A. Masoumi, D. Mehta, E. J. Weinberg, and X. Xiao, Tumbling through a landscape: Evidence of instabilities in high-dimensional moduli spaces, Phys. Rev. D 88, 026005 (2013).

[16] N. Turok, On quantum tunneling in real time, New J. Phys. 16, 063006 (2014).

[17] See Supplemental Material at http://link.aps.org/ supplemental/10.1103/PhysRevLett.123.031601 for a brief derivation of our approach as an expansion of the full quantum dynamics.

[18] S. Mrowczynski and B. Muller, Wigner functional approach to quantum field dynamics, Phys. Rev. D 50, 7542 (1994).

[19] E. P. Wigner, On the quantum correction for thermodynamic equilibrium, Phys. Rev. 40, 749 (1932). 
[20] C. Gardiner and P. Zoller, Quantum noise, a handbook of Markovian and non-Markovian quantum stochastic methods with applications to quantum optics (Springer, Berlin, 2004).

[21] P. W. Anderson, Absence of diffusion in certain random lattices, Phys. Rev. 109, 1492 (1958).

[22] D. Podolsky and K. Enqvist, Eternal inflation and localization on the landscape, J. Cosmol. Astropart. Phys. 02 (2009) 007.

[23] J. Braden, J. R. Bond, and L. Mersini-Houghton, Cosmic bubble and domain wall instabilities I: parametric amplification of linear fluctuations, J. Cosmol. Astropart. Phys. 03 (2015) 007.

[24] J. C. Butcher, Implicit Runge-Kutta processes, Math. Comput. 18, 50 (1964).

[25] L. Kofman, A. D. Linde, and A. A. Starobinsky, Reheating after Inflation, Phys. Rev. Lett. 73, 3195 (1994).

[26] L. Kofman, A. D. Linde, and A. A. Starobinsky, Towards the theory of reheating after inflation, Phys. Rev. D 56, 3258 (1997).

[27] S. Yu. Khlebnikov and I. I. Tkachev, Classical decay of inflaton, Phys. Rev. Lett. 77, 219 (1996).

[28] G. N. Felder and I. Tkachev, LATTICEEASY: A Program for lattice simulations of scalar fields in an expanding universe, Comput. Phys. Commun. 178, 929 (2008).

[29] J. Berges, S. Borsanyi, and C. Wetterich, Prethermalization, Phys. Rev. Lett. 93, 142002 (2004).
[30] A. D. Linde, Decay of the false vacuum at finite temperature, Nucl. Phys. B216, 421 (1983); Erratum, Nucl. Phys. B223, 544(E) (1983).

[31] S. Khlebnikov, L. Kofman, A. D. Linde, and I. Tkachev, First-Order Nonthermal Phase Transition after Preheating, Phys. Rev. Lett. 81, 2012 (1998).

[32] S. R. Coleman, Quantum tunneling and negative eigenvalues, Nucl. Phys. B298, 178 (1988).

[33] J. R. Bond, J. Braden, and L. Mersini-Houghton, Cosmic bubble and domain wall instabilities III: The role of oscillons in three-dimensional bubble collisions, J. Cosmol. Astropart. Phys. 09 (2015) 004.

[34] D. Polarski and A. A. Starobinsky, Semiclassicality and decoherence of cosmological perturbations, Classical Quantum Gravity 13, 377 (1996).

[35] C. Kiefer, D. Polarski, and A. A. Starobinsky, Quantum to classical transition for fluctuations in the early universe, Int. J. Mod. Phys. D 07, 455 (1998).

[36] C. P. Burgess, R. Holman, G. Tasinato, and M. Williams, EFT beyond the horizon: Stochastic inflation and how primordial quantum fluctuations go classical, J. High Energy Phys. 03 (2015) 090.

[37] E. Nelson, Quantum decoherence during inflation from gravitational nonlinearities, J. Cosmol. Astropart. Phys. 03 (2016) 022.

[38] W. H. Zurek, Decoherence, einselection, and the quantum origins of the classical, Rev. Mod. Phys. 75, 715 (2003). 